Countering beam divergence effects with focused segmented scintillators for high DQE megavoltage active matrix imagers

This article has been downloaded from IOPscience. Please scroll down to see the full text article.

2012 Phys. Med. Biol. 575343

(http://iopscience.iop.org/0031-9155/57/16/5343)

View the table of contents for this issue, or go to the journal homepage for more

Download details:

IP Address: 141.211.173.82

The article was downloaded on $26 / 06 / 2013$ at $14: 46$

Please note that terms and conditions apply. 


\title{
Countering beam divergence effects with focused segmented scintillators for high DQE megavoltage active matrix imagers
}

\author{
Langechuan Liu, Larry E Antonuk ${ }^{1}$, Qihua Zhao, Youcef El-Mohri \\ and Hao Jiang \\ Department of Radiation Oncology, University of Michigan, Ann Arbor, MI 48109, USA \\ E-mail: antonuk@med.umich.edu
}

Received 3 February 2012, in final form 27 June 2012

Published 1 August 2012

Online at stacks.iop.org/PMB/57/5343

\begin{abstract}
The imaging performance of active matrix flat-panel imagers designed for megavoltage imaging (MV AMFPIs) is severely constrained by relatively low x-ray detection efficiency, which leads to a detective quantum efficiency (DQE) of only $\sim 1 \%$. Previous theoretical and empirical studies by our group have demonstrated the potential for addressing this constraint through the utilization of thick, two-dimensional, segmented scintillators with optically isolated crystals. However, this strategy is constrained by the degradation of high-frequency DQE resulting from spatial resolution loss at locations away from the central beam axis due to oblique incidence of radiation. To address this challenge, segmented scintillators constructed so that the crystals are individually focused toward the radiation source are proposed and theoretically investigated. The study was performed using Monte Carlo simulations of radiation transport to examine the modulation transfer function and DQE of focused segmented scintillators with thicknesses ranging from 5 to $60 \mathrm{~mm}$. The results demonstrate that, independent of scintillator thickness, the introduction of focusing largely restores spatial resolution and DQE performance otherwise lost in thick, unfocused segmented scintillators. For the case of a $60 \mathrm{~mm}$ thick BGO scintillator and at a location $20 \mathrm{~cm}$ off the central beam axis, use of focusing improves DQE by up to a factor of $\sim 130$ at non-zero spatial frequencies. The results also indicate relatively robust tolerance of such scintillators to positional displacements, of up to $10 \mathrm{~cm}$ in the source-to-detector direction and $2 \mathrm{~cm}$ in the lateral direction, from their optimal focusing position, which could potentially enhance practical clinical use of focused segmented scintillators in MV AMFPIs.
\end{abstract}

(Some figures may appear in colour only in the online journal)

1 Author to whom any correspondence should be addressed. 


\section{Introduction}

In modern external beam radiotherapy, the goal of maximizing dose to the tumor, while minimizing dose to surrounding normal tissues, is assisted through routine imaging of the megavoltage (MV) treatment beam immediately prior to, and during, treatment (i.e. irradiation) of the patient. The electronic portal imaging devices (EPIDs) presently employed to perform such imaging are typically based on the technology of active matrix flat-panel imagers (AMFPIs) (Antonuk 2004). AMFPIs that are configured for MV imaging consist of an indirect detection, active matrix array of pixels coupled to an x-ray converter consisting of a relatively thick rare-earth phosphor screen and an overlying metal plate (e.g., $\sim 133 \mathrm{mg} \mathrm{cm}^{-2}$ of $\mathrm{Gd}_{2} \mathrm{O}_{2} \mathrm{~S}$ :Tb with a thickness of $\sim 360 \mu \mathrm{m}$ and $\sim 1 \mathrm{~mm}$ of copper) (Antonuk et al 1992, El-Mohri et al 2001, Antonuk 2002, 2004). In addition, an electronic acquisition system controls the operation and readout of the array. In such conventional MV AMFPIs, incident x-rays interact with the converter, resulting in the deposition of energy and the creation of light photons in the screen. Some of these photons exit the screen and are detected by optical sensors (i.e. photodiodes) in the pixels, resulting in the generation and storage of the imaging signal. This signal is subsequently read out by the acquisition electronics, resulting in the formation of a digital image. Due to their many advantages (Antonuk 2002), MV AMFPIs have largely replaced portal film and other earlier EPID technologies, and are currently regarded as the gold standard for portal imaging (Antonuk 2002, Pouliot et al 2005).

While the usefulness of conventional MV AMFPIs has made these devices practically ubiquitous in radiotherapy environments, their performance is strongly constrained by inefficient use of the incident radiation. Specifically, conventional MV AMFPIs detect only $\sim 2 \%$ of incoming $\mathrm{x}$-rays at $6 \mathrm{MV}$, leading to a maximum detective quantum efficiency (DQE) of only $\sim 1 \%$ (El-Mohri et al 2001). This pales in comparison with maximum DQE values of over $70 \%$ for AMFPIs designed for diagnostic imaging at kilovoltage energies (Marshall et al 2011). Increasing the efficiency of $x$-ray detection through the use of thicker x-ray converters (e.g., based on thicker phosphor screens) (Wowk and Shalev 1994) will not generally result in DQE improvement due to spatial resolution loss caused by the increased spread of optical photons. In response to this challenge, many strategies have been explored to increase DQE performance-with various prototypes based on area (Mosleh-Shirazi et al 1998, Seppi et al 2003, Sawant et al 2005a, Maltz et al 2009) and linear (Keller et al 2002, Rathee et al 2006, Samant and Gopal 2006, Kirvan et al 2010) detectors providing DQE values as high as $\sim 9 \%$ and $20 \%$, respectively. (In addition, an innovative linear scanning system based on a double row of discrete $\mathrm{ZnWO}_{4}$ crystals provided an x-ray quantum efficiency (QE) of $\sim 50 \%$ (Morton et al 1991).) One strategy that has shown increasing promise in recent empirical and theoretical investigations involves replacement of the phosphor screen with a thick, crystalline, segmented scintillator (Sawant et al 2005b, 2006, Wang et al 2008, 2009a, 2009b, El-Mohri et al 2011). Segmented scintillators consist of a two-dimensional matrix of high-aspectratio elements in the form of scintillating crystals separated by septal wall material (e.g., polystyrene) which limits lateral spread of optical photons (Wang et al 2009a). In addition, compared to a phosphor screen, the superior optical transparency of crystalline scintillator material limits the deterioration of DQE due to optical Swank noise (Sawant et al 2005a, Wang et al 2009a). These properties allow segmented scintillators to achieve progressively higher DQE performance with increasing thickness, while limiting the loss of spatial resolution due to optical blur. For example, a prototype MV AMFPI incorporating an $\sim 11 \mathrm{~mm}$ thick segmented $\mathrm{BGO}\left(\mathrm{Bi}_{4} \mathrm{Ge}_{3} \mathrm{O}_{12}\right)$ scintillator with an element-to-element pitch of $1016 \mu \mathrm{m}$ has been shown to provide a maximum DQE value of $\sim 20 \%$, which is $\sim 20$ times greater than that of a conventional MV AMFPI (Wang et al 2009b). Such performance improvement has 

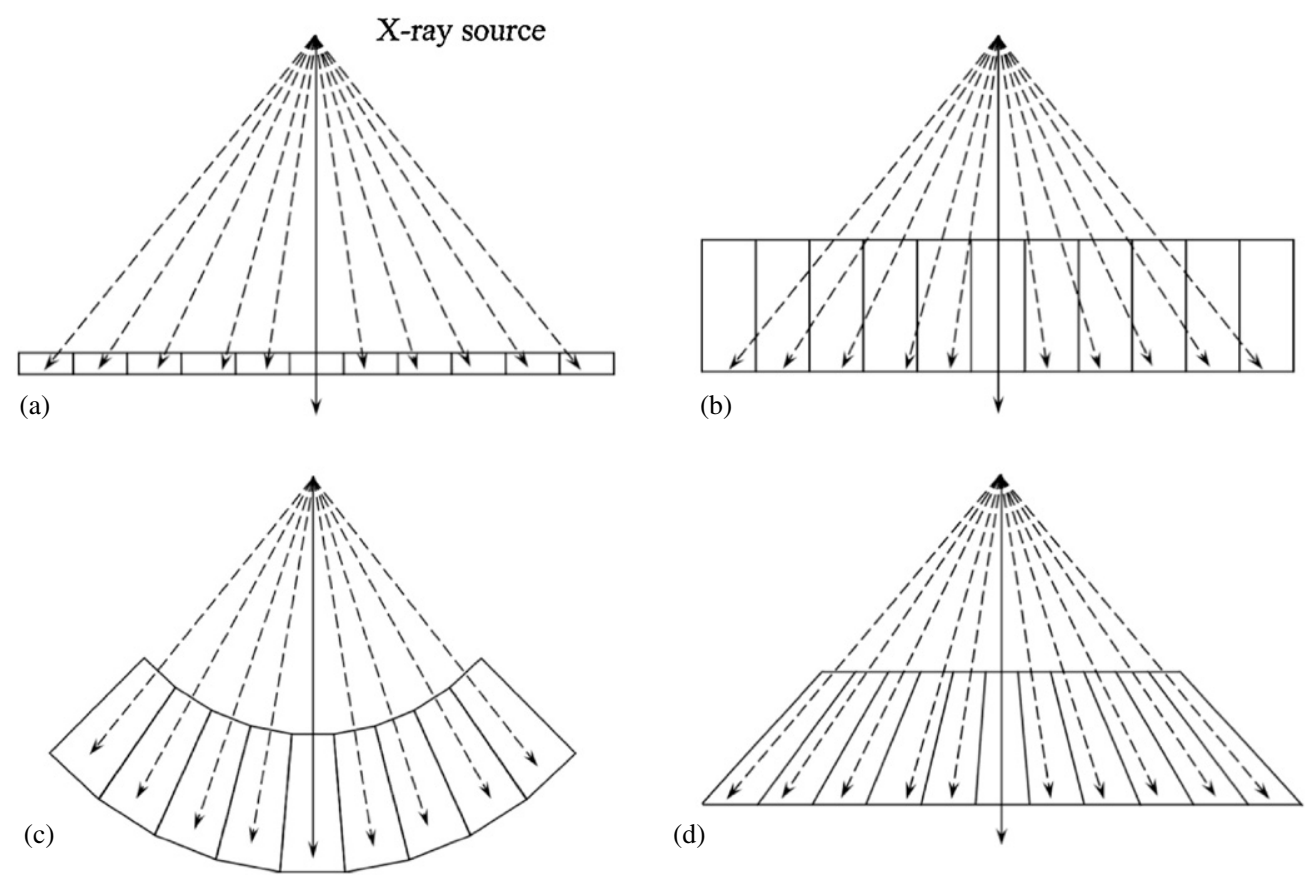

Figure 1. (a, b) Schematic illustration (not to scale) of the problematic effect of geometric beam divergence. X-rays following a given track (black dashed arrows) away from the central beam axis (black solid arrows) can deposit energy in different neighboring scintillating crystals, leading to loss of spatial resolution-an effect that becomes more pronounced with increasing scintillator thickness, as illustrated in (a) and (b). A schematic cross-sectional view of two geometric approaches for addressing beam divergence: (c) a curved, focused scintillator geometry; and (d) a planar, focused scintillator geometry. Note that, for purposes of clarity of illustration, the metal plate typically positioned over the scintillator is not shown.

enabled the acquisition of high-quality portal images at extremely low doses, down to one beam pulse per image. Moreover, the acquisition of high-quality MV cone beam CT images at a total scan dose of only $\sim 4$ cGy-comparable to the dose required to obtain a single portal image from a conventional MV AMFPI-has also been demonstrated (El-Mohri et al 2011). From the resulting reconstructed CT images, differences in electron density as small as $\sim 2.76 \%$ have been delineated.

Given such encouraging results obtained from earlier investigations of the segmented scintillator strategy, it is of interest to explore the imaging performance of thicker scintillators that allow even more efficient use of MV radiation. In particular, for scintillator thicknesses greater than $\sim 10 \mathrm{~mm}$, spatial resolution (quantified by the modulation transfer function (MTF)) and DQE performance are increasingly compromised by geometric beam divergence (Wang et al 2010). This effect originates from the oblique angle of incidence of primary $\mathrm{x}$-rays originating from the source with respect to the sidewalls of a segmented scintillator at locations away from the central beam axis. As a result, primary x-rays following a given track can deposit energy in different neighboring elements depending on the point of interaction across the thickness of the scintillator-resulting in spatial resolution loss and DQE degradation. As illustrated in figures 1(a) and (b), this effect becomes increasingly pronounced at locations progressively farther from the central beam axis, as well as for progressively thicker scintillators. 

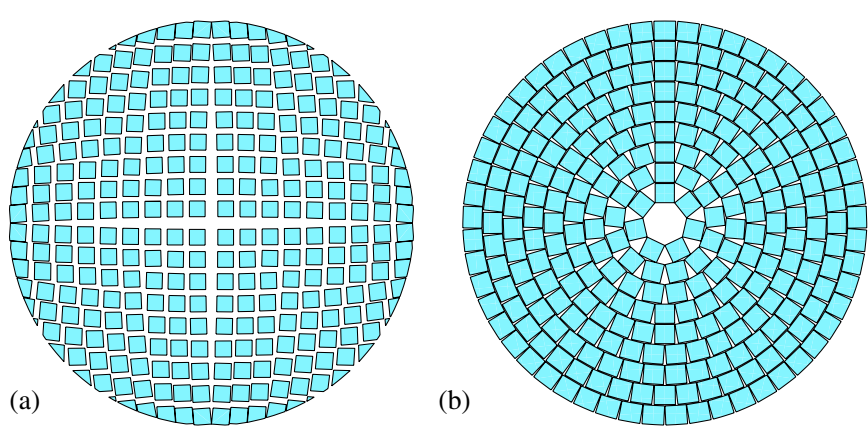

(c)

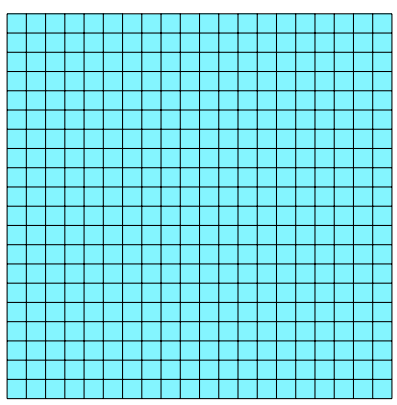

Figure 2. Top views of two possible arrangements of individual scintillating crystals on a spherical cap: (a) a warped rectangular arrangement and (b) a concentric ring arrangement-both of which correspond to the curved, focused geometry of figure 1(c). (c) The top view of a rectilinear grid arrangement of individual scintillating crystals in the absence of septal wall materialcorresponding to the planar, focused geometry of figure 1(d).

In order to counter the performance degrading effect of beam divergence, the individual scintillating crystals (as well as their septal walls) should be constructed so as to be focused toward the radiation source. Illustrations of two different geometric approaches that satisfy this requirement are shown in figures $1(\mathrm{c})$ and $(\mathrm{d})$.

Figure 1(c) illustrates the conceptually simple approach of employing a curved geometry in which the top and bottom surfaces of the scintillator have the shape of concentric spherical caps with a common virtual center located at the position of the radiation source. In one embodiment of this geometry, every crystal has the same shape and is focused toward, and positioned at the same distance from, the radiation source. The use of a single crystal shape across such a curved geometry offers the possible advantage of relatively straightforward crystal fabrication. Two possible arrangements of the crystals for such an embodiment, each employing a different crystal shape, are illustrated in figures 2(a) and (b). In figure 2(a), a warped rectangular arrangement in which the crystals are positioned along two sets of perpendicularly intersecting longitudinal lines on the spherical cap is shown. (Note that the crystals are truncated in the peripheral region.) In figure 2(b), a concentric ring arrangement in which the crystals are positioned along a series of latitudinal lines with equal angular separation is shown. Both designs provide a scintillating crystal occupancy (which shall be referred to as a fill factor) of less than $100 \%$, since it is impossible to tessellate a spherical cap with thousands of crystals of the same single shape (White et al 1992). More importantly, the designs in figures 2(a) and (b) have a variable element-to-element pitch (defined as the distance between the center of two adjacent elements), which would significantly complicate registration between the elements and the pixels in an underlying imaging array. In addition, the spherical bottom surface of such scintillators favors the use of an imaging array having the same curved shape-precluding the use of existing flat-panel array technology which employs rigid substrates (Antonuk 2002, 2004). While a prototype active matrix imaging array fabricated on a flexible substrate and configured into an approximately hemispherical shape has been demonstrated (Street et al 2009), the development of such imaging arrays is only in its infancy, and the registration of scintillators consisting of thousands of crystals with such curved arrays would present formidable challenges.

An alternative to the spherical cap approach, involving a planar geometry, is shown in figure $1(\mathrm{~d})$ in which both the top and bottom surfaces of the scintillator are flat and parallel to each other. The methods required to fabricate specific designs of such focused planar geometries are expected to require adjustment to the techniques (Sawant et al 2006) presently 
used to construct unfocused segmented prototypes. However, the rectilinear grid arrangement of scintillating crystals illustrated in figure 2(c) provides a fill factor higher than those of curved designs in the presence of septal walls of the same minimum width-as discussed in appendix A. Moreover, this geometry offers the considerable advantage of a fixed elementto-element pitch. The flat bottom surface and the fixed pitch of scintillator designs based on this approach would facilitate a straightforward registration with existing AMFPI arrays. Overall, given the probable practical advantages in creating MV AMFPIs employing converters based on the focused, planar, segmented scintillator approach, this study concentrates on an exploration of the theoretical performance of such converters.

\section{Methods}

\subsection{Monte Carlo simulations}

The MTF, noise power spectrum (NPS) and DQE performance of a variety of hypothetical MV x-ray converter designs incorporating segmented scintillators were examined through the Monte Carlo simulation of radiation transport. Optical transport of photons generated in the scintillators was not included in this study due to the fact that beam divergence is expected to be primarily determined by radiation transport and also due to the prohibitively large computational time, the inclusion of optical transport would have required. The simulations were performed using the EGSnrc code, a package widely employed for medical physics applications (Kawrakow and Rogers 2000). Simulations were performed on a variety of focused and unfocused designs. In the simulations, the geometric shapes of the designs were modeled using the EGSnrc C++ class library (egspp) (Kawrakow 2005). The user code, as well as the geometry configuration input file, was modified, as necessary, to facilitate the present studies.

The simulations employed a point x-ray source with a $6 \mathrm{MV}$ spectrum obtained through interpolation of a tabulated spectrum file corresponding to the energy spectrum of a Varian LINAC (Sheikh-Bagheri 1999). Cutoff energies for photons and electrons were set to 0.01 and $0.521 \mathrm{MeV}$, respectively_both corresponding to a kinetic energy of $10 \mathrm{keV}$. Other simulation parameters were set to the values used in a previous segmented scintillator study (Wang et al 2010). Using a 64-bit Linux CPU cluster with 100, $1.8 \mathrm{GHz}$ AMD Opteron processors, a total of $\sim 142000$ CPU hours were required to perform the simulations.

\subsection{Overview of converter designs}

For the hypothetical converters investigated in this study, segmented scintillator thicknesses, $T_{\text {sci }}$, of 5, 10, 20, 40 and $60 \mathrm{~mm}$, coupled to an overlying $1 \mathrm{~mm}$ thick copper plate, were simulated. In an earlier theoretical study of the effects of beam divergence on unfocused segmented scintillators (Wang et al 2010), incoming radiation was modeled as a parallel rectangular beam tilted at different incident angles to quantify those effects in unfocused converter designs. In the present study, a point radiation source is employed to more accurately model the physical situation. Use of even more realistic extended sources was found to result in negligible (i.e. less than 1\%) changes to MTF for both unfocused and focused converters compared to a point source. In addition, both focused and unfocused converter designs are examined - to investigate the effectiveness of focused designs in addressing the imaging performance degradation due to beam divergence. Conceptually, as illustrated in figure 3 , the converters were considered to have a $40 \times 40 \mathrm{~cm}^{2}$ detection area, centered at the central beam axis of the radiation field and situated at a source-to-detector distance (SDD) of $130 \mathrm{~cm}$, 


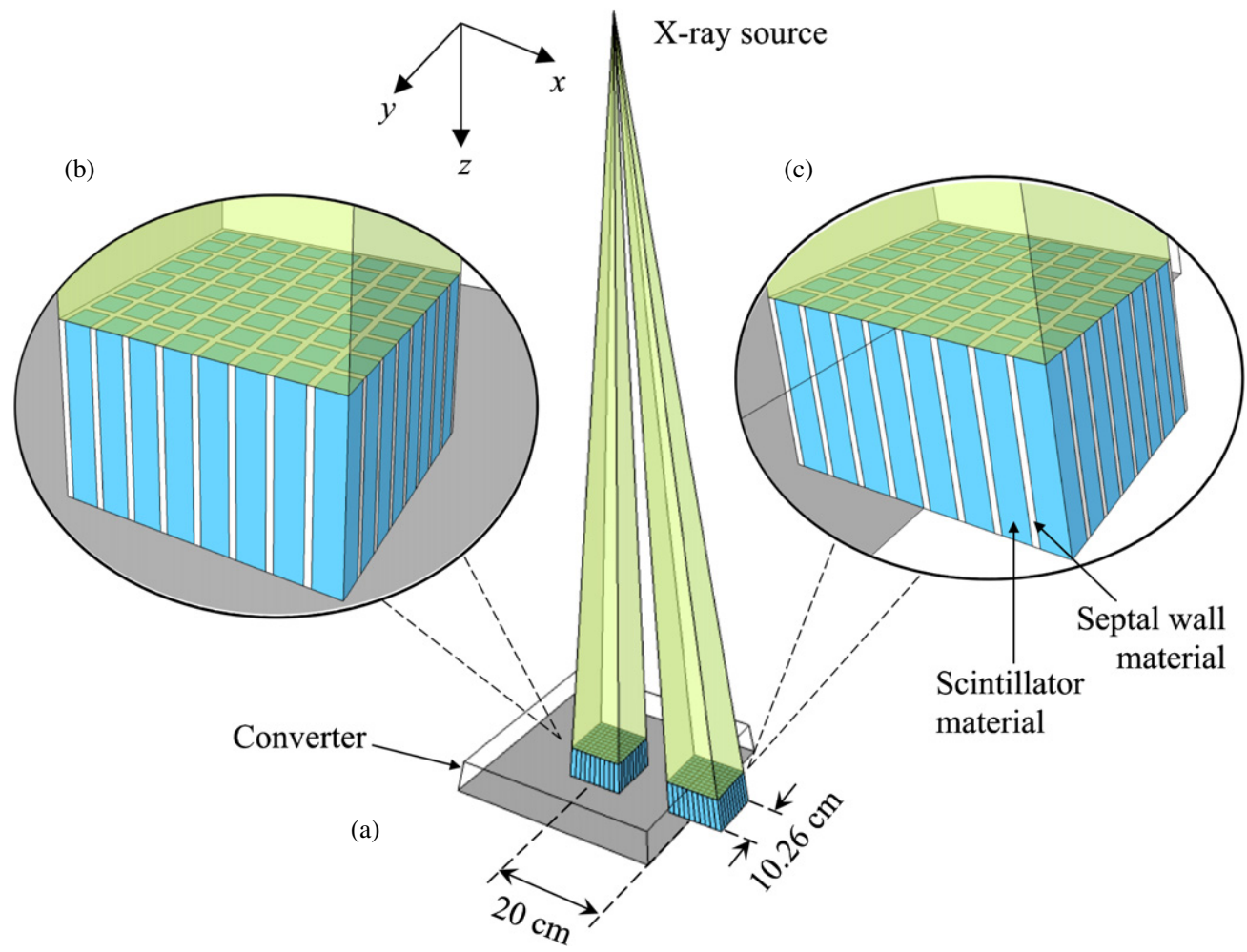

Figure 3. (a) Schematic illustration of the geometric setup employed in the radiation transport simulations. The converter (depicted as a transparent wire frame with a shaded bottom surface) incorporates a thick, focused, segmented scintillator design. Two simulated regions, each consisting of a sub-matrix of scintillator elements (comprising scintillator and septal wall material), are portrayed at the central beam axis and $20 \mathrm{~cm}$ off-axis at the periphery of the converter. As illustrated in magnified insets (b) and (c) corresponding to these regions, those scintillator elements located toward the periphery of the converter are more obliquely shaped. Note that the sub-matrix shown in (c) extends beyond the edge of the converter, so as to facilitate evaluation of imaging performance near the periphery. The transparent volumes delineate the space traversed by x-rays that are emitted from the source and reach the top surface of each selected region.

reflecting the approximate size and positioning of typical clinical MV AMFPIs. In order to evaluate the performance of the converters for increasingly oblique incident angles, selected regions of the converter located progressively further away (i.e. at distances of $0,5,10$, 15 and $20 \mathrm{~cm}$ ) from the central beam axis were modeled. Each of these simulated regions consists of a sub-matrix of $101 \times 101$ scintillator elements (corresponding to an area of $10.26 \times 10.26 \mathrm{~cm}^{2}$ at the bottom surface of the scintillator) centered at the designated location. This area is sufficiently large to allow accurate local determination of frequency dependent metrics, while small enough to allow the simulations to be performed within manageable times. For example, the NPS simulation for a single region required between $\sim 120$ and $2700 \mathrm{CPU}$ hours (corresponding to elapsed run times of $\sim 1.2$ to $27 \mathrm{~h}$ ), depending upon the design of the converter.

For the focused designs, each element is a square frustum (i.e. a truncated pyramid) with the virtual apex located at the point radiation source. Within a given quadrant of the converter, the shape of every frustum is different in order to accommodate the changing obliqueness of the incident radiation. However, the square bottom bases of all the individual frusta are 
Table 1. Physical properties (Knoll 2010, Bell 2012) of the various scintillator materials examined in this study. Note that the decay times listed for $\mathrm{CsI}$ and $\mathrm{CdWO}_{4}$ consist of two components with corresponding weightings indicated in the brackets.

\begin{tabular}{llllll}
\hline Material & Chemical formula & $\begin{array}{l}\text { Light output } \\
\left(\text { photons } \mathrm{keV}^{-1}\right)\end{array}$ & Decay time $(\mu \mathrm{s})$ & $\begin{array}{l}\text { Physical } \\
\text { density } \\
\left(\mathrm{g} \mathrm{cm}^{-3}\right)\end{array}$ & $\begin{array}{l}\text { Electron } \\
\text { density } \\
\left(\mathrm{mol} \mathrm{cm}^{-3}\right)\end{array}$ \\
\hline $\mathrm{CsI}$ & $\mathrm{CsI}: \mathrm{Tl}$ & 64.8 & $0.68(64 \%)$, & 4.51 & 1.87 \\
$\mathrm{BGO}$ & $\mathrm{Bi}_{4} \mathrm{Ge}_{3} \mathrm{O}_{12}$ & 8.2 & $3.34(36 \%)$ & & 7.13 \\
$\mathrm{LYSO}$ & $\mathrm{Lu}_{1.8} \mathrm{Y}_{0.2} \mathrm{SiO}_{4}: \mathrm{Ce}$ & 32 & 0.3 & 7.10 & 3.04 \\
$\mathrm{CdWO}$ & $\mathrm{CdWO}_{4}$ & 15 & 0.04 & 7.9 & 3.38 \\
\end{tabular}

geometrically identical, resulting in uniform pitch at the bottom surface of the segmented scintillator. This pitch was chosen to be $1016 \mu \mathrm{m}$ (with $100 \mu \mathrm{m}$ polystyrene septal walls and $916 \mu \mathrm{m}$ scintillator crystals) in both orthogonal directions-corresponding to the dimensions and wall composition of several prototypes previously reported by our group (Sawant et al 2006). Since the shapes of the top and bottom bases are geometrically similar, the pitch at the top surface of the scintillator is also uniform, and is reduced by a factor of $\varphi$ compared to that at the bottom surface, where

$$
\varphi=\frac{\mathrm{SDD}}{\mathrm{SDD}+T_{\mathrm{sci}}} .
$$

It is interesting to note that, for a focused converter of a given thickness and element pitch, the ability of elements to detect incident x-rays varies slowly as a function of position across the converter. While the volume of the elements, as well as the fill factor of the elements (i.e. the fraction of element volume occupied by scintillator material), remains constant throughout the converter (see appendix B), the efficiency of detection of incident x-rays (i.e. the $\mathrm{x}$-ray $\mathrm{QE}$ ) increases for elements more distant from the center of the converter as a result of the increasing radiation path length for those elements. As a result, elements located on the periphery of a $40 \times 40 \mathrm{~cm}^{2}$ converter are $\sim 0.9 \%-0.3 \%$ more efficient in detecting incident radiation compared to elements near the center of the converter for thicknesses ranging from 5 to $60 \mathrm{~mm}$.

In the simulations, four candidate scintillator materials (CsI, BGO, LYSO and $\mathrm{CdWO}_{4}$ ) were examined. These materials were selected on the basis of their desirable properties which include high physical and electron densities, high optical output, and short decay time, as summarized in table 1. For each of the CsI and BGO materials, a total of ten converter designs (corresponding to five scintillator thicknesses for each of the focused and unfocused geometries) were modeled, while fewer designs were modeled for $\mathrm{LYSO}$ and $\mathrm{CdWO}_{4}$.

\subsection{Determination of MTF}

As shown in figure 4(a), each MTF (and NPS) simulation was performed on a portion of the converter located at a distance $d_{x}$ from the central beam axis. MTF was determined by calculating the one-dimensional Fourier transform of a line spread function (LSF) obtained using the angled slit technique (Fujita et al 1992, Dobbins 2000). In the simulations, the slit was modeled as a $10.26 \mathrm{~cm} \times 4 \mu \mathrm{m}$ area, defining an aperture for the incident $\mathrm{x}$-rays. The longer dimension of this area was positioned at a $2^{\circ}$ tilt angle relative to the $y$-axis, as schematically illustrated in figure 4(b). Energy deposited in each scintillating crystal within the region of interest was plotted as a function of the distance between the center of the elements 


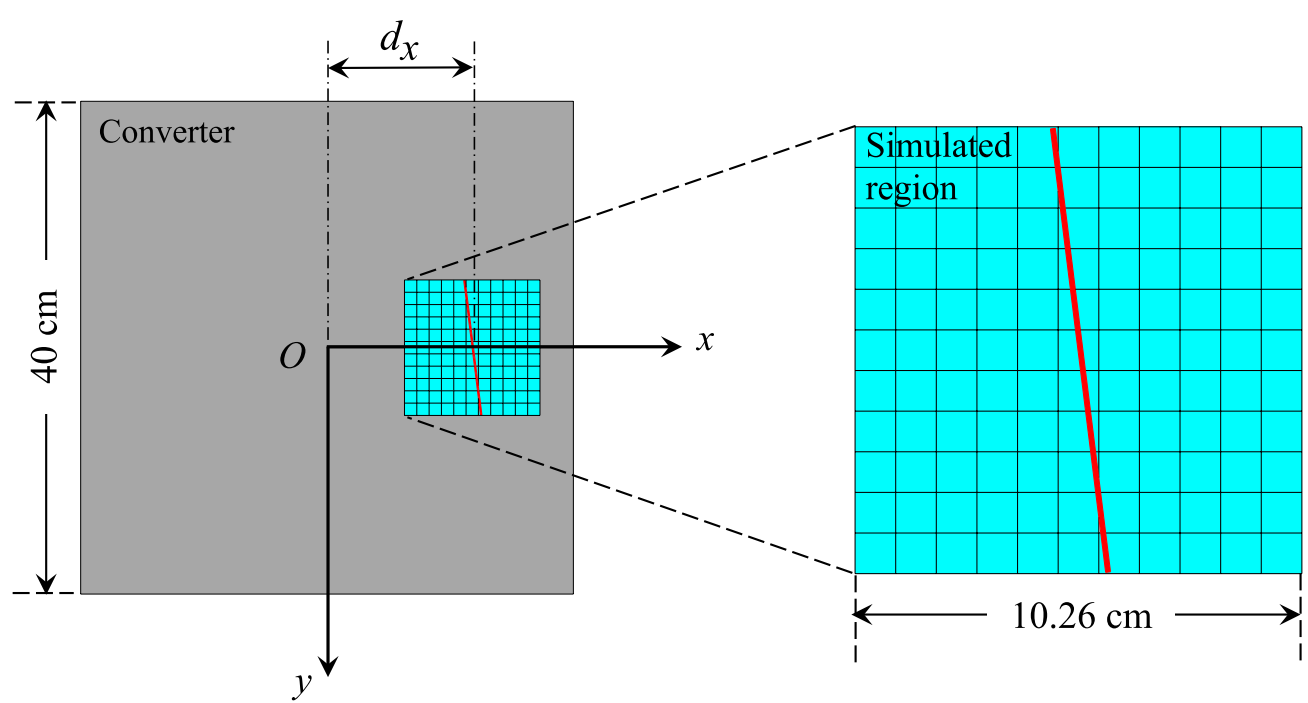

(a)

(b)

Figure 4. (a) Schematic illustration of the simulation geometry used in the determination of MTF and NPS. The grid represents a simulated region of the converter (indicated in solid gray). For the case of the MTF simulations, the angled line corresponds to the slit area which forms a $2^{\circ}$ angle with respect to the $y$-axis. In this study, simulations were performed at various regions located at distances, $d_{x}$, ranging from 0 to $20 \mathrm{~cm}$ from the central beam axis, $O$. (b) A magnified view of the simulated portion of the converter.

and the tilted slit, resulting in the LSF. For each simulated region of each converter design, $4 \times 10^{5}$ primary $\mathrm{x}$-ray histories were employed.

\subsection{Determination of normalized NPS (NNPS) and DQE}

NPS was obtained from the Fourier transform of zero-mean data realizations, using the synthesized slit technique (Giger et al 1984, Maidment and Yaffe 1994, Sawant et al 2005b). For each simulated region of each converter design, a total of 400 simulations were performed with ten million primary $\mathrm{x}$-ray histories per simulation, resulting in 400 frames. For each frame, only the central $81 \times 81$ elements (leaving a ten-element-wide margin on each side) were used and summed along one direction, providing a realization with 81 points. For each realization, the average of the 81 points was subtracted from the value for each point, generating a zeromean realization, upon which a one-dimensional Fourier transform was performed. NPS was obtained by averaging the 400 Fourier transforms obtained from the 400 frames, and NNPS was then determined from the equation given below (Wang et al 2010):

$$
\operatorname{NNPS}(f)=\frac{\bar{q}_{0} \operatorname{NPS}(f)}{\bar{A}^{2}},
$$

where $\bar{q}_{0}$ is the incident $\mathrm{x}$-ray fluence, and $\bar{A}$ is the average energy deposited in each scintillating crystal. Finally, DQE was calculated from the simulation results for MTF and NNPS using the equation given below (Cunningham 2000):

$$
\operatorname{DQE}(f)=\frac{\operatorname{MTF}^{2}(f)}{\operatorname{NNPS}(f)}
$$



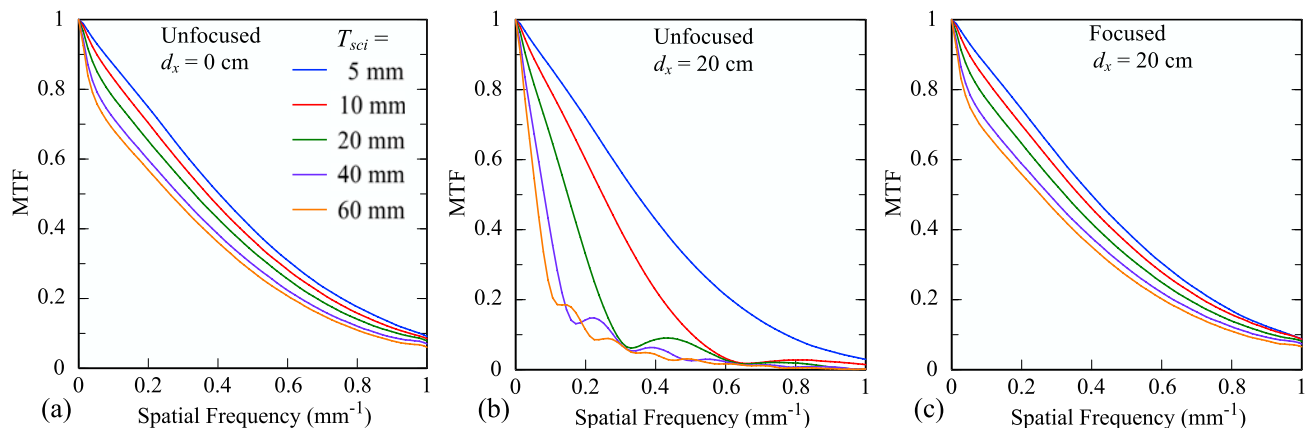

Figure 5. MTF results for converters incorporating segmented BGO scintillators with thicknesses, $T_{\text {sci }}$, ranging from 5 to $60 \mathrm{~mm}$ for: (a) unfocused designs at a location corresponding to the central beam axis, (b) unfocused designs at $20 \mathrm{~cm}$ off-axis and (c) focused designs at $20 \mathrm{~cm}$ off-axis.

\section{Results}

\subsection{Modulation transfer function}

Simulation results for the MTF performance for converter designs incorporating segmented BGO scintillators with thicknesses ranging from 5 to $60 \mathrm{~mm}$ are shown in figure 5 . For unfocused converters, MTF determined at the central beam axis (where beam divergence effects are negligible) degrades with increasing thickness, as seen in figure 5(a). For example, at a spatial frequency of $0.49 \mathrm{~mm}^{-1}$ (corresponding to the Nyquist frequency associated with the $1016 \mu \mathrm{m}$ pitch of the elements), MTF drops from $\sim 0.4$ to $\sim 0.3$. This degradation of spatial resolution for thicker scintillators is mainly due to the increasing lateral spread of energy deposited by Compton electrons, as well as the higher probability of re-absorption of recoil Compton x-rays. For regions located further away from the central beam axis, beam divergence effects become progressively more pronounced for thicker, unfocused scintillators, as illustrated in figure 5(b) which shows results corresponding to $20 \mathrm{~cm}$ off-axis. (Note that the first minimum in the results for thicker scintillators appears at increasingly lower frequencies, which correspond closely to the lateral displacement created by the projection of the possible points of interaction across the thickness of the scintillator (Wang et al 2010).) At $0.49 \mathrm{~mm}^{-1}$, the MTF results for the $5 \mathrm{~mm}$ thickness exhibit only a slight degradation from 0.4 at the central beam axis to $\sim 0.3$ at $20 \mathrm{~cm}$ off-axis. By comparison, the MTF results for the $60 \mathrm{~mm}$ thickness decrease dramatically from $\sim 0.3$ at the central beam axis to less than 0.05 at $20 \mathrm{~cm}$ off-axis. Such a significant drop in MTF largely nullifies the advantage of greater attenuation provided by thicker segmented scintillators. However, as demonstrated in figure 5(c), for converter designs incorporating focused segmented scintillators, MTF values $20 \mathrm{~cm}$ off-axis are restored to levels very close to (i.e. within 0.01 ) the central beam axis values shown in figure 5(a).

MTF results for converters incorporating unfocused and focused $60 \mathrm{~mm}$ thick BGO segmented scintillators at various locations away from the central beam axis are shown in figure 6. For the unfocused converter, MTF decreases at locations increasingly farther from the central beam axis, as shown in figure 6(a). Such variation of MTF would result in blurrier images in regions further away from the central axis. For the focused converter, the MTF is observed to be nearly independent of location, as is evident by the almost completely overlapping curves in figure 6(b), indicating high and uniform spatial resolution across the entire $40 \times 40 \mathrm{~cm}^{2}$ converter. 

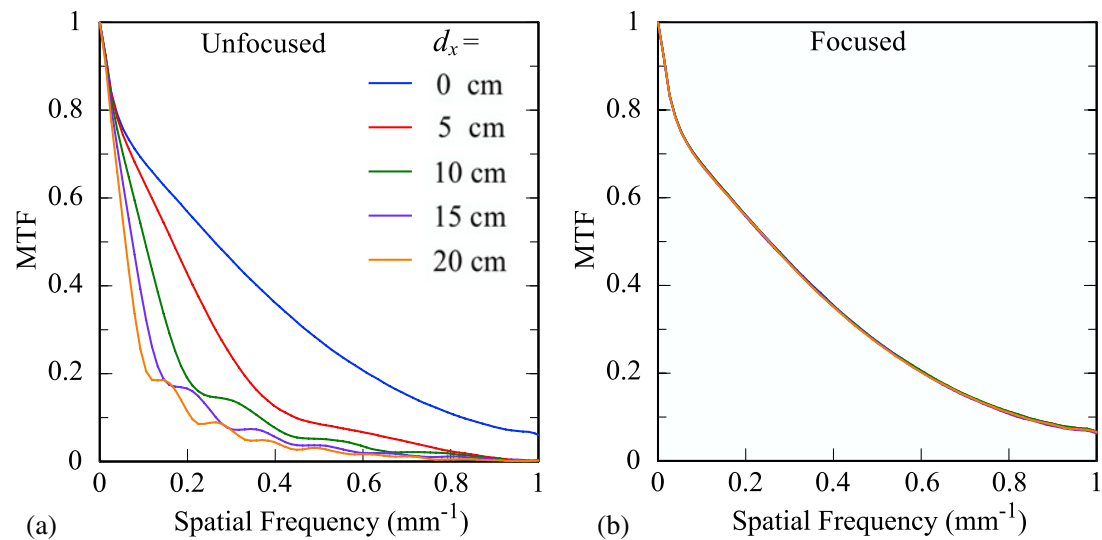

Figure 6. MTF results for converters incorporating $60 \mathrm{~mm}$ thick BGO scintillators at locations, $d_{x}$, ranging from 0 to $20 \mathrm{~cm}$ away from the central beam axis for (a) unfocused and (b) focused designs.
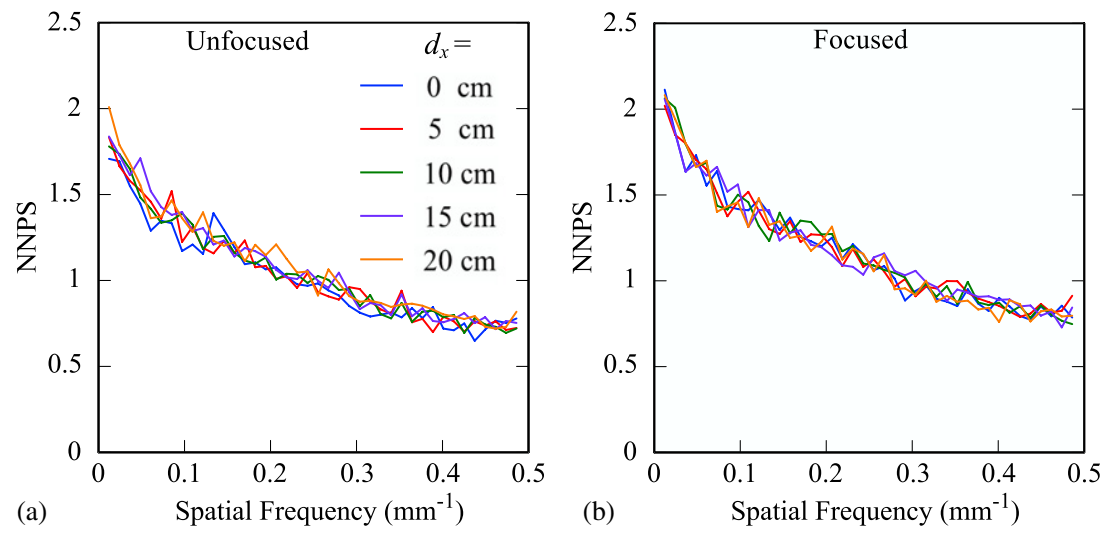

Figure 7. NNPS results for two converters incorporating $60 \mathrm{~mm}$ thick BGO scintillators at locations, $d_{x}$, ranging from 0 to $20 \mathrm{~cm}$ away from the central beam axis for (a) unfocused and (b) focused designs.

\subsection{Normalized NPS}

NNPS performance for two converters incorporating unfocused and focused $60 \mathrm{~mm}$ thick BGO segmented scintillators for various locations away from the central beam axis is shown in figure 7. For the converter employing the unfocused scintillator, NNPS for the various regions largely overlap, as shown in figure 7(a). This invariance in NNPS is due to the largely unchanged size and shape of the energy deposition of secondary Compton electrons over the range of incident x-ray angles employed in the simulations (Wang et al 2010). For the converter employing the focused scintillator, while NNPS for the various regions also largely overlaps (for the same reasons as above), the results are systematically larger than those for the unfocused converter-as seen in figure 7(b). This difference is due to the lower $\mathrm{x}$-ray detection efficiency of the focused converter ( $\sim 6 \%$ less for a $60 \mathrm{~mm}$ BGO scintillator) that is a consequence of the good alignment of the less attenuating septal wall material with $\mathrm{x}$-ray trajectories from the source. 


\subsection{Detective quantum efficiency}

DQE performance for converter designs incorporating segmented BGO scintillators with various thicknesses is shown in figure 8. For unfocused converters and at the central beam axis, DQE increases with scintillator thickness for all frequencies up to the Nyquist frequency, as seen in figure 8(a). However as seen in figure 8(b), at $20 \mathrm{~cm}$ off-axis and compared with thinner scintillators, although converters with thicker unfocused scintillators have greater lowfrequency DQE values, they suffer from faster and steeper drop in DQE at high frequencies due to more severe beam divergence effects. As a consequence, beyond certain frequencies, DQE values for thicker unfocused scintillators are even lower than those of thinner scintillators. For example, for the converter with a $60 \mathrm{~mm}$ thick unfocused scintillator, the DQE value decreases significantly from over 0.40 at zero frequency to $\sim 0.04$ at $0.1 \mathrm{~mm}^{-1}$. This decrease in DQE is mainly due to the severe drop in MTF at high frequencies shown in figure 6(a). However, as can be seen in figure $8(\mathrm{c})$, with the introduction of focusing, DQE values at $20 \mathrm{~cm}$ off-axis are restored to within 0.10 of their levels without focusing at the central beam axis (figure 8(a)), as would be expected given the corresponding MTF and NNPS behaviors observed in figures 6(b) and 7(b), respectively. This is a significant restoration-representing, in the example of a $60 \mathrm{~mm}$ thick BGO scintillator, an increase in DQE of up to a factor of $\sim 130$ compared to the corresponding unfocused scintillator.

DQE results for converters incorporating unfocused and focused $60 \mathrm{~mm}$ thick BGO segmented scintillators at various locations are shown in figure 9. For the unfocused converter, DQE exhibits a dramatic decrease at locations progressively further away from the central beam axis, as shown in figure 9(a). Such variation in DQE, which is due to MTF loss for locations off-axis, would lead to a progressive degradation of image quality further away from the central beam axis. For the focused converter, DQE curves are independent of location, as is evident in figure 9(b) - which should lead to a greatly improved and more uniform image quality across a $40 \times 40 \mathrm{~cm}^{2}$ converter.

\subsection{Simulation of converters based on other scintillator materials}

Imaging performance at $20 \mathrm{~cm}$ off-axis for converter designs incorporating $60 \mathrm{~mm}$ thick, focused scintillators based on various scintillator materials is shown in figure 10. As seen in figure $10(\mathrm{a})$, the MTF values for BGO, LYSO and $\mathrm{CdWO}_{4}$ converters largely overlap, providing similar spatial resolution. This can be attributed to a similar degree of lateral spread of deposited energy as a result of the similar electron densities (and therefore radiation stopping powers) of these materials. Note that the slightly lower MTF of the CsI converter is a result of reduced capacity to limit lateral spread of Compton electrons due to a lower electron density. For the same reason (i.e. lower electron density and thus lower x-ray detection efficiency), the CsI converter exhibits higher NNPS values than those based on the other three scintillator materials which almost overlap, as shown in figure 10(b). Therefore, as expected from equation (3), converter designs with $\mathrm{BGO}$, LYSO and $\mathrm{CdWO}_{4}$ scintillators provide similar DQE performance that is higher than that for CsI.

\subsection{Tolerance of focused converter designs to imager displacement}

A given focused planar converter design will generally be constructed for a specific position (e.g., for a specified SDD and for no lateral displacement with respect to the central beam axis) so as to ensure optimal focusing and imaging performance over the entire converter. Displacement of the converter from that intended position would be expected to lead to defocusing, resulting in some degree of degradation of spatial resolution and DQE 

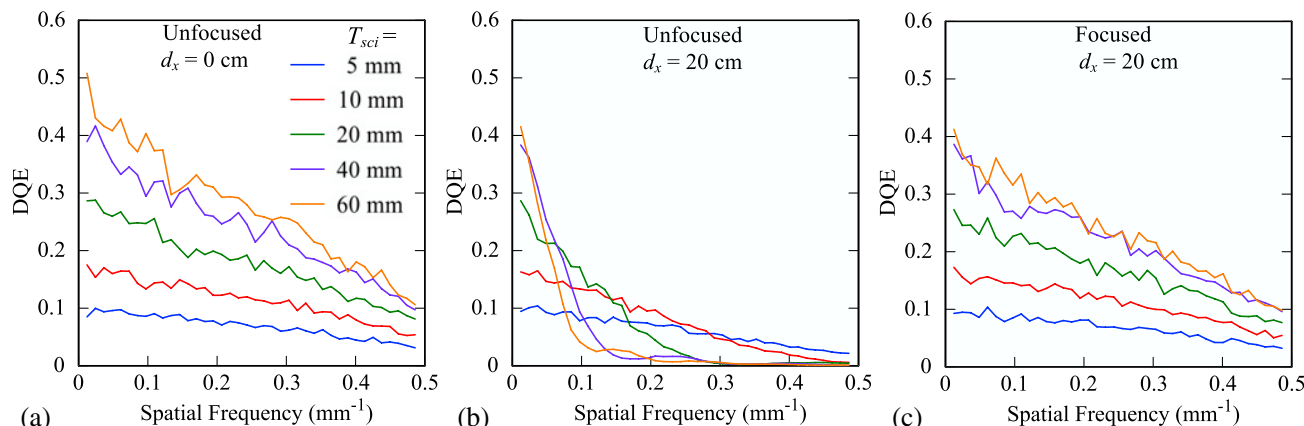

Figure 8. DQE results for converters incorporating segmented BGO scintillators with thicknesses, $T_{\text {sci }}$, ranging from 5 to $60 \mathrm{~mm}$ for: (a) unfocused designs at a location corresponding to the central beam axis, (b) unfocused designs at $20 \mathrm{~cm}$ off-axis and (c) focused designs at $20 \mathrm{~cm}$ off-axis.
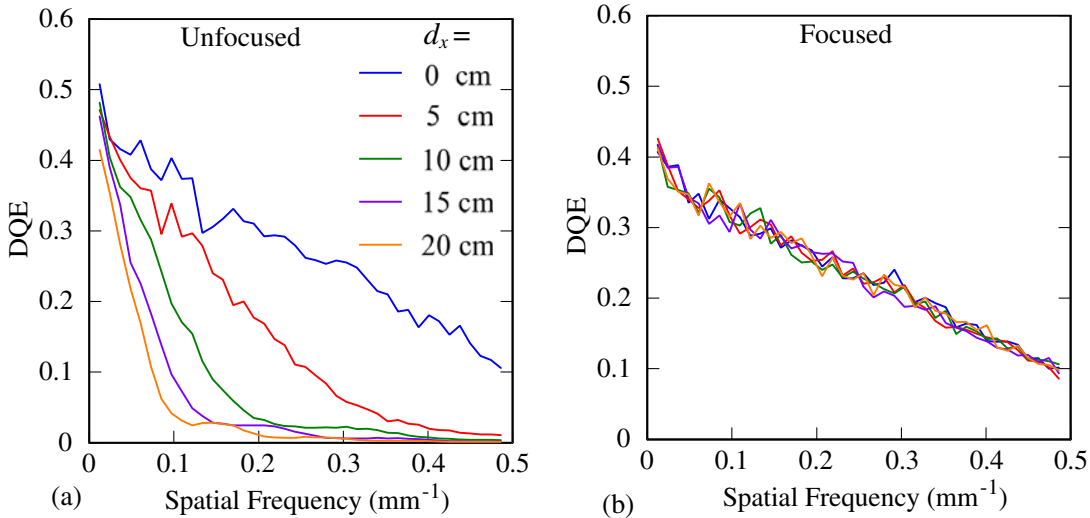

Figure 9. DQE results for converters incorporating $60 \mathrm{~mm}$ thick BGO scintillators at locations, $d_{x}$, ranging from 0 to $20 \mathrm{~cm}$ away from the central beam axis for (a) unfocused and (b) focused designs.
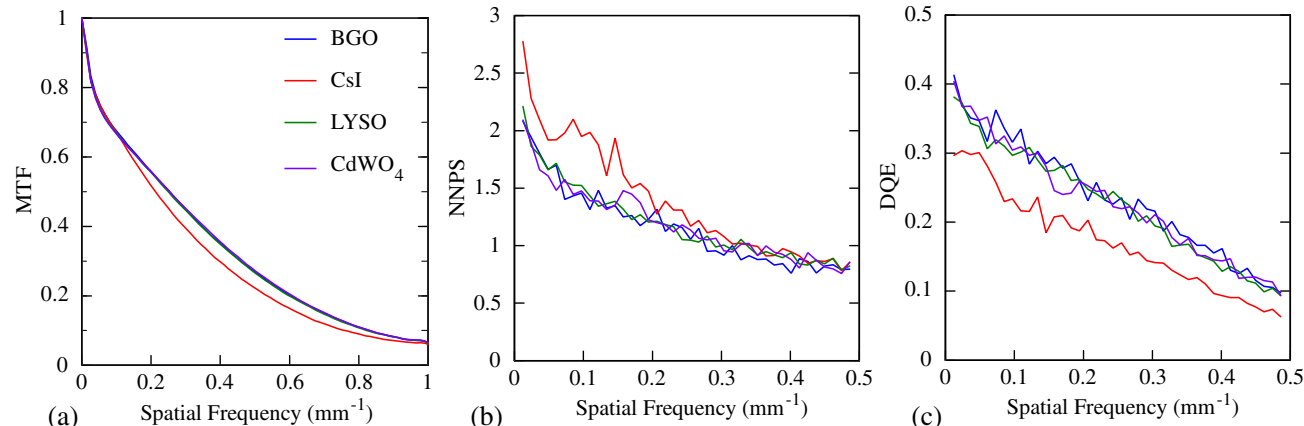

Figure 10. Simulation results at $20 \mathrm{~cm}$ off-axis for focused converters incorporating $60 \mathrm{~mm}$ thick scintillators consisting of various scintillator materials for: (a) MTF, (b) NNPS and (c) DQE.

performance. To quantify the effect of operating a focused converter at non-optimal positions, simulations were performed for a converter incorporating a $60 \mathrm{~mm}$ thick BGO segmented scintillator designed for optimal performance at an SDD of $130 \mathrm{~cm}$, as a function of SDD 


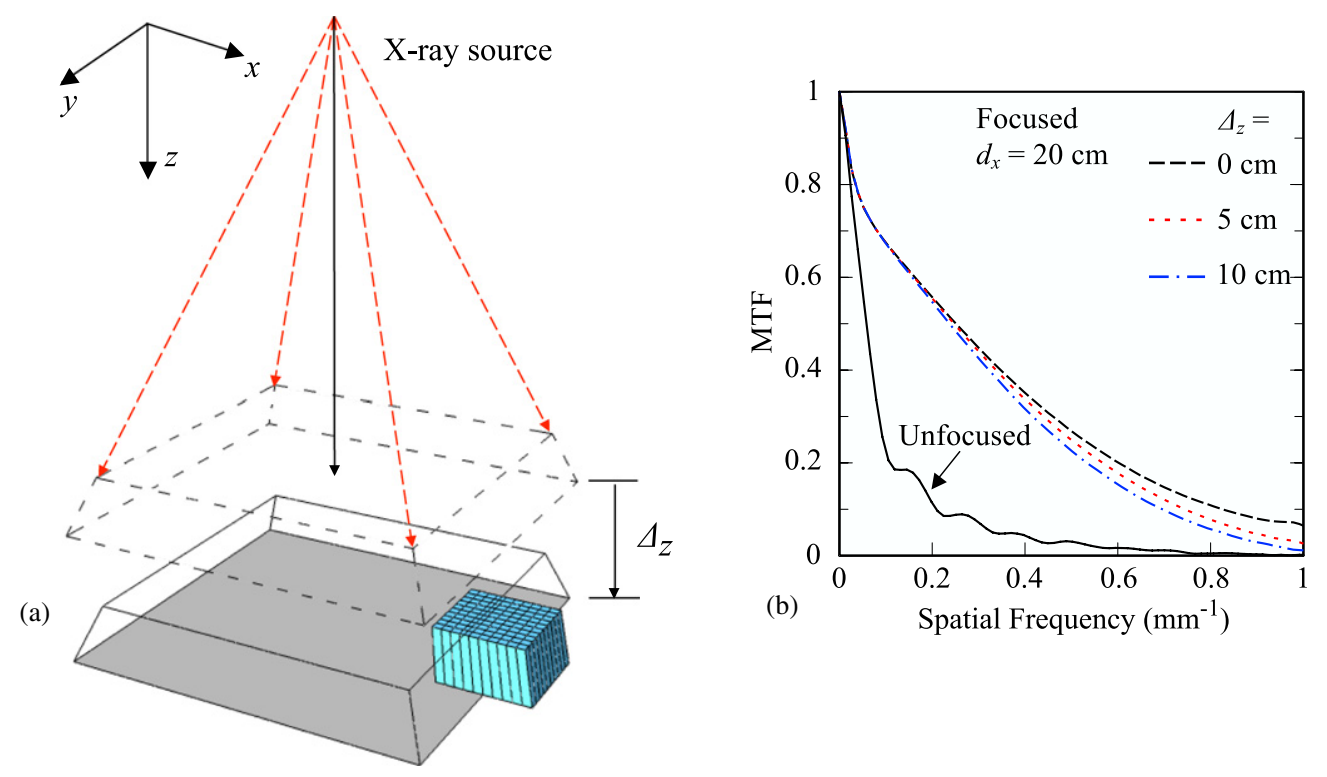

Figure 11. (a) Schematic drawing corresponding to simulations in which a converter (represented as a wire frame with a shaded bottom surface) is displaced by a distance $\Delta_{z}$ along the source-todetector ( $z$-)direction from the optimal focusing position (dashed wire frame). In these simulations, the converter has no lateral displacement and is therefore centered at the central beam axis (solid arrow). The simulated region (portrayed as a segmented volume) is located $20 \mathrm{~cm}$ off-axis. (b) MTF results for that region, for a focused converter with a $60 \mathrm{~mm}$ thick BGO scintillator, for various $\Delta_{z}$ displacements. For comparison, the MTF determined at $20 \mathrm{~cm}$ off-axis for an unfocused converter with a $60 \mathrm{~mm}$ thick BGO scintillator (with no SDD displacement) is shown by the solid line.

(z-direction) and lateral ( $x$-direction) displacements, as schematically illustrated in figures 11(a) and 12(a), respectively. Figure 11(b) shows the MTF performance at $20 \mathrm{~cm}$ off-axis, for SDD displacements progressively further away from the radiation source. It can be seen that MTF suffers only slight degradation, even for a $10 \mathrm{~cm}$ SDD displacement, and still provides much higher performance than that of an unfocused converter. Note that while these MTF results are for displacements in the positive $z$-direction, results for displacements in the negative $z$-direction (i.e. closer to the radiation source) exhibit almost identical values and thus are not shown. Figure 12(b) shows the MTF performance at $20 \mathrm{~cm}$ off-axis for various lateral displacements. The MTF degradation caused by a $2 \mathrm{~cm}$ lateral displacement is found to be very similar to that caused by a $10 \mathrm{~cm}$ SDD displacement.

These results suggest that the MTF performance of focused converters is more sensitive to lateral displacement than SDD displacement, as would perhaps be expected, given the geometric construction of the focused elements. However, the results also indicate that, even when focused converters are operated relatively far from their optimal focusing position (i.e. up to at least $10 \mathrm{~cm}$ in the SDD direction or $2 \mathrm{~cm}$ in the lateral direction), they still provide significantly improved spatial resolution compared to that of unfocused converters. Note that NNPS was found to remain essentially unchanged for all displacements considered and, thus, DQE degradation can largely be attributed to MTF loss. The relatively small sensitivity of imager performance to SDD and lateral displacement could be of practical interest if it were clinically useful to allow such displacement capability so as to accommodate unusual clinical situations-e.g., larger SDD to accommodate particularly obese patients or lateral displacement to view far-off-axis regions of large fields. 
(a)

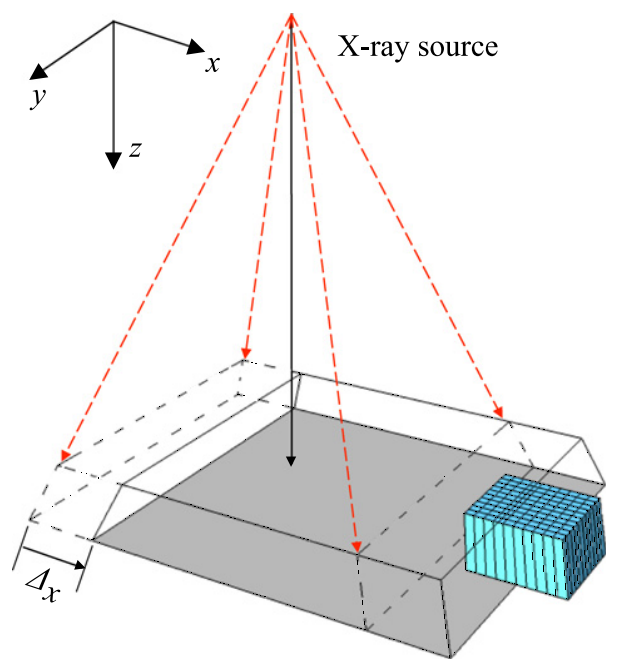

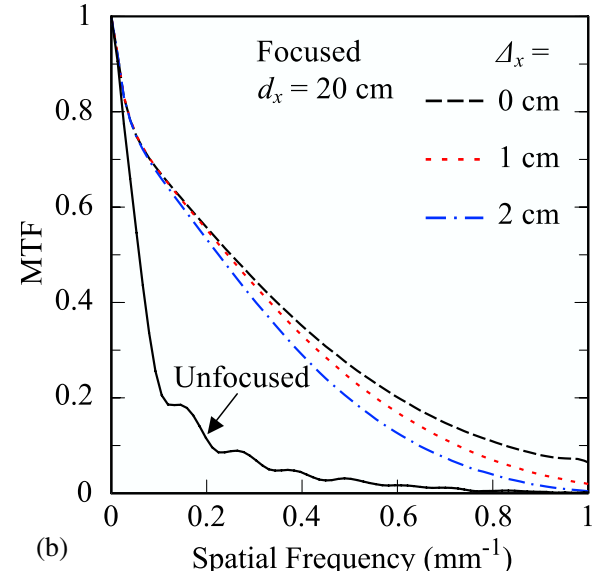

(b)

Figure 12. (a) Schematic drawing corresponding to simulations in which a converter is displaced a distance $\Delta_{x}$ in a lateral ( $x$-)direction from the optimal focusing position (dashed wire frame). In these simulations, the converter has no SDD displacement. The simulated region (portrayed as a segmented volume) is located $20 \mathrm{~cm}$ away from the center of the converter. (b) MTF results for that region, for a focused converter with a $60 \mathrm{~mm}$ thick BGO scintillator, for various $\Delta_{x}$ displacements. For comparison, the MTF determined at $20 \mathrm{~cm}$ off-axis for an unfocused converter with a $60 \mathrm{~mm}$ thick BGO scintillator is shown by the solid line.

\section{Summary and discussion}

The results reported in this paper strongly suggest that the degradation of imaging performance of thick converters caused by beam divergence can be largely eliminated through the introduction of planar, focused segmented scintillators-at least for thicknesses up to $60 \mathrm{~mm}$. Moreover, the performance of the focused planar converter designs examined in this study is only weakly degraded by moderate SDD or lateral displacement from the optimal focusing position. Based on these encouraging results, further investigations on focused prototypes are underway. For example, as a natural extension to the current study, optical transport simulations will be carried out to explore the optical properties of focused designs, including the effect of element-to-element pitch on optical Swank noise.

\section{Acknowledgment}

This project is supported by NIH grant R01 CA051397.

\section{Appendix A}

For the warped rectangular and concentric ring arrangements of individual scintillating crystals on a spherical cap illustrated in figures 2(a) and (b), respectively, the fill factors are both less than $100 \%$. Moreover, the fill factors for these arrangements of a curved, focused scintillator geometry would vary with the solid angles subtended by each crystal and by the entire converter. For the example of a converter positioned at a distance of $130 \mathrm{~cm}$ from the $\mathrm{x}$-ray source, with a base diameter of $40 \mathrm{~cm}$, a crystal cross-sectional dimension of $1016 \times 1016 \mu \mathrm{m}^{2}$, and with no minimum septal wall thickness, the fill factors would be, at most, $97.2 \%$ (rectangular) and $94.3 \%$ (ring). However, the presence of septal walls having a minimum thickness would 
cause further reduction in fill factor. For the aforementioned setup, a crystal cross-sectional dimension of $966 \times 966 \mu \mathrm{m}^{2}$ and a minimum septal wall thickness of $50 \mu \mathrm{m}$ lead to fill factors of $87.9 \%$ (rectangular) and $85.3 \%$ (ring), while a $916 \times 916 \mu \mathrm{m}^{2}$ crystal and a minimum septal wall thickness of $100 \mu \mathrm{m}$ lead to fill factors of $79.0 \%$ (rectangular) and $76.7 \%$ (ring).

By comparison, for the rectilinear grid arrangement of individual scintillating crystals of a focused, planar scintillator geometry, as illustrated in figure 2(c), while the nominal fill factor would be $100 \%$, the fill factor would decrease with the introduction of septal walls. For example, for elements with a pitch of $1016 \mu \mathrm{m}$, the fill factor would be reduced to $90.4 \%$ and $81.3 \%$ for wall thicknesses of 50 and $100 \mu \mathrm{m}$, respectively, independent of the area of the overall scintillator. Thus, for comparable element sizes and septal wall thicknesses, the fill factor of the rectilinear grid arrangement is greater than that of warped rectangular and concentric ring arrangements discussed above.

\section{Appendix B}

For the rectilinear grid arrangement of a planar, focused segmented scintillator geometry such as schematically illustrated in figures 1(d) and 3, the individual elements have the shape of a frustum and the volume of each element is given by

$$
V=\frac{h}{3}\left(A_{t}+A_{b}+\sqrt{A_{t} A_{b}}\right),
$$

where $h$ is the perpendicular height of the frustum, $A_{t}$ and $A_{b}$ are the areas of the top and bottom surfaces of the frustum, respectively, and $A_{t}$ is always less than $A_{b}$. Since the elementto-element pitch on the top and bottom surfaces remains constant across the scintillator, $A_{t}$ and $A_{b}$ also remain constant. In addition, all the elements share the same perpendicular height $h$ (which is equal to the scintillator thickness). Therefore, according to equation (B.1), all the elements for a given rectilinear grid arrangement have the same volume, despite the variation in shape of those elements across the scintillator.

For each frustum-shaped element, although the cross-sectional area of the scintillator crystal and that of the entire element varies along the thickness of the scintillator (both becoming smaller going from the bottom to the top surface), the ratio of these two areas remains constant and is equal to the fraction of entire element volume occupied by the scintillator crystal (i.e. the fill factor). In the case of a converter with an element pitch of $1016 \mu \mathrm{m}$ on the bottom surface and $100 \mu \mathrm{m}$ septal walls, the fill factor of an element is $\sim 81.3 \%$, as given by $\left(\frac{1016-100}{1016}\right)^{2}$, across the entire converter.

\section{References}

Antonuk L E 2002 Electronic portal imaging devices: a review and historical perspective of contemporary technologies and research Phys. Med. Biol. 47 R31-65

Antonuk LE 2004 Thin Film Transistors, Materials and Processes, Volume 1: Amorphous Silicon Thin Film Transistors ed Y Kuo (Boston: Kluwer) pp 395-484

Antonuk L E, Boudry J, Huang W, McShan D L, Morton E J, Yorkston J, Longo M J and Street R A 1992 Demonstration of megavoltage and diagnostic $\mathrm{X}$-ray imaging with hydrogenated amorphous silicon arrays Med. Phys. 19 1455-66

Bell Z W 2012 Handbook of Particle Detection and Imaging ed C Grupen and I Buvat (Berlin: Springer) pp 349-75

Cunningham I A 2000 Handbook of Medical Imaging ed J Beutel et al (Bellingham, WA: SPIE Optical Engineering Press) pp 79-160

Dobbins J T 2000 Handbook of Medical Imaging ed J Beutel et al (Bellingham, WA: SPIE Optical Engineering Press) pp 163-222

El-Mohri Y, Antonuk L E, Zhao Q, Choroszucha R B, Jiang H and Liu L 2011 Low-dose megavoltage cone-beam CT imaging using thick, segmented scintillators Phys. Med. Biol. 56 1509-27 
El-Mohri Y, Jee K-W, Antonuk L E, Maolinbay M and Zhao Q 2001 Determination of the detective quantum efficiency of a prototype, megavoltage indirect detection, active matrix flat-panel imager Med. Phys. 28 2538-50

Fujita H, Tsai D Y, Itoh T, Doi K, Morishita J, Ueda K and Ohtsuka A 1992 A simple method for determining the modulation transfer function in digital radiography IEEE Trans. Med. Imaging 11 34-9

Giger M L, Doi K and Metz C E 1984 Investigation of basic imaging properties in digital radiography: 2. Noise Wiener spectrum Med. Phys. 11 797-805

Kawrakow I 2005 egspp: the EGSnrc C++ class library Technical Report PIRS-899 National Research Council of Canada, Ottawa, Canada

Kawrakow I and Rogers D W O 2000 The EGSnrc code system: Monte Carlo simulation of electron and photon transport Technical Report PIRS-701 National Research Council of Canada, Ottawa, Canada

Keller H, Glass M, Hinderer R, Ruchala K, Jeraj R, Olivera G and Mackie T R 2002 Monte Carlo study of a highly efficient gas ionization detector for megavoltage imaging and image-guided radiotherapy Med. Phys. 29 165-75

Kirvan P F, Monajemi T T, Fallone B G and Rathee S 2010 Performance characterization of a MVCT scanner using multislice thick, segmented cadmium tungstate-photodiode detectors Med. Phys. 37 249-57

Knoll G F 2010 Radiation Detection and Measurement (New Jersey: Wiley)

Maidment A D A and Yaffe M J 1994 Analysis of the spatial-frequency-dependent DQE of optically coupled digital mammography detectors Med. Phys. 21 721-9

Maltz J, Dubouloz A, Paidi A, Gangadharan B, Hartmann J, Bani-Hashemi A and Hoerauf G 2009 Thick monolithic pixelated scintillator array for megavoltage imaging - Conference Abstract Med. Phys. 36 2818-9

Marshall N W, Mackenzie A and Honey I E 2011 Quality control measurements for digital X-ray detectors Phys. Med. Biol. 56 979-99

Morton E J, Swindell W, Lewis D G and Evans P M 1991 A linear array, scintillation crystal-photodiode detector for megavoltage imaging Med. Phys. 18 681-91

Mosleh-Shirazi M A, Evans P M, Swindell W, Symonds-Tayler J R N, Webb S and Partridge M 1998 Rapid portal imaging with a high-efficiency, large field-of-view detector Med. Phys. 25 2333-46

Pouliot J et al 2005 Low-dose megavoltage cone-beam CT for radiation therapy Int. J. Radiat. Oncol. Biol. Phys. 61 552-60

Rathee S, Tu D, Monajemi T T, Rickey D W and Fallone B G 2006 A bench-top megavoltage fan-beam CT using CdWO4-photodiode detectors: I. System description and detector characterization Med. Phys. 33 1078-89

Samant S S and Gopal A 2006 Analysis of the kinestatic charge detection system as a high detective quantum efficiency electronic portal imaging device Med. Phys. 33 3557-67

Sawant A, Antonuk L E, El-Mohri Y, Zhao Q, Wang Y, Li Y, Du H and Perna L 2006 Segmented crystalline scintillators: Empirical and theoretical investigation of a high quantum efficiency EPID based on an initial engineering prototype $\mathrm{CsI}(\mathrm{Tl})$ detector Med. Phys. 33 1053-66

Sawant A et al 2005a Segmented phosphors: MEMS-based high quantum efficiency detectors for megavoltage x-ray imaging Med. Phys. 32 553-65

Sawant A et al 2005b Segmented crystalline scintillators: an initial investigation of high quantum efficiency detectors for megavoltage x-ray imaging Med. Phys. 32 3067-83

Seppi E J et al 2003 Megavoltage cone-beam computed tomography using a high-efficiency image receptor Int. J. Radiat. Oncol. Biol. Phys. 55 793-803

Sheikh-Bagheri D 1999 Monte Carlo study of photon beams from medical linear accelerators: optimization, benchmark and spectra PhD Dissertation Department of Physics, Carleton University, Ottawa, Canada

Street R A, Wong W S and Lujan R 2009 Curved electronic pixel arrays using a cut and bend approach J. Appl. Phys. 105104504

Wang Y, Antonuk L E, El-Mohri Y and Zhao Q 2009a A Monte Carlo investigation of Swank noise for thick, segmented, crystalline scintillators for radiotherapy imaging Med. Phys. 36 3227-38

Wang Y, Antonuk L E, El-Mohri Y, Zhao Q, Sawant A and Du H 2008 Monte Carlo investigations of megavoltage cone-beam CT using thick, segmented scintillating detectors for soft tissue visualization Med. Phys. 35 145-58

Wang Y, Antonuk L E, Zhao Q, El-Mohri Y and Perna L 2009b High-DQE EPIDs based on thick, segmented BGO and CsI:Tl scintillators: performance evaluation at extremely low dose Med. Phys. 36 5707-18

Wang Y, El-Mohri Y, Antonuk L E and Zhao Q 2010 Monte Carlo investigations of the effect of beam divergence on thick, segmented crystalline scintillators for radiotherapy imaging Phys. Med. Biol. 55 3659-73

White D, Kimerling J A and Overton S W 1992 Cartographic and geometric components of a global sampling design for environmental monitoring Cartography Geogr. Inform. Sci. 19 5-22

Wowk B and Shalev S 1994 Thick phosphor screens for on-line portal imaging Med. Phys. 21 1269-76 